\title{
Zur Anthropologie Calvins Menschenwürde - Imago dei Zwischen Humanistischem und Theologischem Ansatz
}

\author{
HANS HELMUT ESSER
}

Calvins Antropologie darstellen, heisst seine Theologie darstellen und umgekehrt, ohne dass für ihn die verhängnisvolle Einbahnstrasse gölte, von Gott reden hiesse nur vom Menschen reden. Wegen der im Eingang der Institutio grundgelegten und von der Theologie her bestimmten Wechselbeziehung ist es sachlich unangemessen, Calvins Anthropologie nach rein formalen Gesichtspunkten an einer Stelle oder aus einigen Abschnitten seines Werkes zu isolieren. Dadurch wird die Aufgabe, eine Anthropologie Calvins zu erstellen, quantitativ so erschwert, dass die Anstrengung der gleichkommt, eine Gesamtdarstellung der Theologie Calvins zu leisten. Suchen wir dennoch nach speziell anthropologischen Akzentuierungen, so bieten sich bekanntlich entlang dem aufriss der institutio folgende Passagen an:

1. Die Möglichkeiten der Gotteserkenntnis der Menschen und ihr Verwirken

2. Die praelapsarische Anthropologie

I, (2) 3-5

3. Die postlapsarische Anthropologie

I, 15

4. Die wahre Menschheit Christi

II, 1-6

5. Die zwei Naturen des Mittlers Christus

II, 12-13

6. Die Wirklichkeit des neuen Menschen

II, 14

III

7. Von den Mitteln der Christusgemeinschaft in Menschengemeinschaft

IV

Der Referent ist dankbar, dass ihm im Rahmen dieses Systems eine prinzipielle Aufgabe von begrenztem Umfang, aber grundlegender Bedeutung zugefallen ist. Das Material zur Imago-Dei-Lehre Calvins ist übersehbar, andererseits haben wir bei der Gottebenbildlichkeitsaussage mit einer theologisch-anthropologischen Grundformel ${ }^{1} \mathrm{zu}$ tun, die für alle status des Menschen von Bedeutung ist und anthropologisch und christologisch Altes und Neues Testament sowie Schöpfung, Soteriologie und Eschatologie miteinander verbindet.

Wir werden versuchen, Auslegung Calvins an den Erkenntnissen heutiger Exegese zu überprüfen, dabei berücksichtend, was an neuen Erkenntnissen in seiner Zeit ihm zufällt bzw. welchen tra- 
ditionellen und zeitbedingten Bedingungen seine Aussagen unterliegen.

Das besondere Interesse soll sich dabei nicht auf die Weiterführung mittelalterlicher Auslegung bei Calvin richten, sondern auf seine Abhängigkeiten von bzw. seine Freiheiten gegenüber humanistischem Denken. Diese Frage ist immer noch schwer zu klären. Erst seit dem Beginn der 70er Jahre hat in Frankreich eine sehr intensive Bemühung um die Erforschung des französischen Humanismus eingesetst, auf deren Ergebnisse die weitere Erforschung der Biographie Calvins weitgehend angewiesen sein wird. Hatte man den beiden bedeutenden Arbeiten zur Sache von T F Torrance ${ }^{2}$ und K Reuter $^{3}$ je verschiedene Einseitigkeiten vorgeworfen - dem einen, Calvin ausschliesslich werkimmanent $\mathrm{zu}$ interpretieren, wenn auch in grossartiger Zusammanschau, dem andern, ihn zu sehr in Abhängigkeit von vorgegebener scotistischer u.a. spätmittelalterlichter, aber auch antiker Begrifflichkeit zu sehen - hat neuerdings die posthum erschienene Monographie von F Wendel über die Beziehung Calvins zum Humanismus ${ }^{4}$ neue Akzente gesetzt, die aufgearbeitet sein wollen.

\section{1. Überprüfung der Auslegung zur Gottebenbildlichkeit des Menschen}

\section{A. Die Kerstelle Gen 1, 26-285}

Calvin bestreitet die traditionelle Unterscheidung zwischen imago als Menschennatur und similitudo als Ausdruck der dieser Natur hinzugefügten Gnadengaben, ferner lehrt er alle weiteren Spekulationen der Kircheväter zu dieser Begriffsunterscheidung ab. Aufgrund von 1,27 und 5,1 erklärt er die Unterscheidung von Bild und Ähnlichkeit für gegenstandlos und sieht den zweiten Ausdruck als wiederholend und verdeutlichend an. Die Kadenz: "Abschwächung einer Gleichheit" 6 wird ihm nicht deutlich - Der Bestimmung der Herrlichkeit des Menschen nähert er sich mit Kategorien des Substanzdenkens. Via negationis werden dabei ausgeschlossen der Leib als Sitz der Imago Dei, seine Dignität als Kunstwerk Gottes. Auch die der Sache am nächsten kommende finktionale Verstehensweise: "Herrschaft über die Erde" (so Chrysostomus) wird ausgeschlossen, aber wenigstens als untergeordneter Aspekt gelten gelassen. - Reformatorisch konsequent setzt er nicht beim mehr direkt zugänglichen Urstande an, sondern beim durch das Evangelium erneuerten und vom Geist wiedergeborenen Menschen. In "rechtschaffener Gerichtigkeit und Heiligkeit" sieht er die Hauptstücke des Ebenbildes. Erst jetzt werden die eigentlich substantiellen Kategorien 
Adams im Urstande, gleichsam im Rückschlussverfahren, eingeführt: "Bild Gottes" bedeutet die Vollkommenheit der ganzen Natur:

1. rechte Einsicht (recta intelligentia)

2. die Triebe unterwarfen sich der Vernunft, (affectus compositos ad rationem)

3. alle Sinne waren gesund und wohlgeordnet (sensus sanos et ordinatos)

4. der erste Adam zeichnete sich in Wahrheit durch alle guten Eigenschaften (bonis omnibus) aus.

In Summa: "der eigentliche Herrschersitz des göttlichen Ebenbildes ist also im Verstand und im Gemüt. "7 Calvin betont dann nich einmal die gleichmässige. Abstimmung der einzelnen Seelenvermögen aufeinander, u.ä.: "Alle Sinne waren fähig und bereit, dem Verstande zu dienen." ${ }^{8}$ Wir haben hier ohne Zweifel mit einer Reihe humanistischer Seinskategorien zu tun des vom Verstande beherrschten, seine Seele in Ausgewogenheit haltenden Menschen, unbeschadet der Tatsache, das Calvin dieses Bild im Jetztstande als "verdunkelt", verzerrt", von "Vernichtung" gezeichnet erklärt, dabei wieder Substanzkategorien verwendend. - In den Erwägungen zur Gleichrangigkeit von Mann und Frau, in Abgrenzung von 1 Kor. 11,7 nähert sich Calvin noch einmal dem Beziehungsdenken: In der menschlichen Natur strahlt Gottes Erhabenheid wider, interpretiert wird sie jedoch an den innerweltlichen Auswirkungen: "indem Verstand, Wille und alle Sinne eine göttliche Ordnung seigen,"9 Der Mandatsauftrag durch Einsetzung, der nach unserer Erkenntnis die "Würde in Beziehung" ausmacht, ${ }^{10}$ hat nach Calvin eher additive Bedeutung: Calvin sagt von ihm: "auch dies gehört zur Würde."11

Was werden wir also ansprechen als "humanistische" Denkweise? Sicherlich das Arbeiten mit "formalen Seinstrukturen". ${ }^{12}$

Sicherlich ebenso das Denken in juristischen Ordnungskategorien, denn Calvin war ja seiner Ausbildung nach primär humanistischer Jurist. Es wird hier nicht möglich und ohnehin objektiv schwierig sein, die Herkunft des Begriffsgebrauchs Calvins Zu orten. Stammen die "humanistischen" Begriffe aus den Bahnen scholastischer Theologie, aus dem Arsenal Augustins, das wiederum sich aus der Antike speiste, oder sind die direkten zeitgenössischen $\mathrm{Hu}$ manismusbeziehungen zu verdanken? Hier ist noch eine gewaltige Konkordanzarbeit erst zu beginnen. Aber schon im Zugang haben wir Kriterien gesammelt, die einzubringen sind: Ein Interesse an detaillierenden anthropologischen Bestimmungen, das auf Substantiierung der Imago gerichtet ist und zugleich die vorgegebene theologische Tradition ablehnt, ein Ordnungs- und Rechtsdenken, das naturrechtlich orientiert scheint: "Der gesunde Verstand (communis sensus) bestätigt, dass es sich um ein unverletzliches Naturgesetz (lex naturae) dabei handle" (zur rechtmässigen Art der Fortpflan- 
zung, V.28) - Auch in Kleinigkeiten der Auslegung kommt humanistischer historisch-kritischer Sinn zum Zuge: die christologische Deutung des "in unserm Bilde", wie sie in der Tradition begegnet, verwirft Calvin, weil sie von "Moses Gedanken weit ab" liege. Auch die positive Entscheidung Calvins liegt auf der Ebene humanistischphilosologischer Deutung: Die Selbstaufforderung Gottes (V. 26) im Wir-Stil lässt auf eine "innere Unterscheidung" in Gott schliessen. Allerdings interpretiert Calvin selbst diese Unterscheidung nicht trinitarisch, sondern er konzediert nur, dass man auch so verfahren könne.

Wir hätten nicht mit der Umsicht Calvins zu tun, kämen die Beziehungsaussagen nicht wenigstens zekundär zum Zuge. In theozentrischer Akzentuierung wird das Beziehungsmoment wieder eingeholt: Der Fortgang der Schöpfung ist Beweis für die Fürsorge für den Menschen. Die "auch" im Bildsein eingeschlossene "Würde", s.o., kommt darin zum Ausdruck, dass der Mensch "feierlich in seine Rechtsstellung eingesetzt wird" (V. 28f) (verum ... iuris sui compos efficitur).

\section{B. Gen $5,1 b-3$}

Calvin setzt hier schon die Bedeutung des Sündenfalls für die Deutung der imago voraus: Die ersten Menschen sind auf einzigartige Weise ins Leben gestellt worden, alle andern stammen aus höherliegender Wurzel und sind von Eltern gezeugt (ex altiori stirpe et parentibus geniti fleurint). ${ }^{13}$ Es sieht zunächst so aus, als komme Calvin zu Beziehungs- und Bestimmungsaussagen, um die Würde der imago festzulegen: Er interpretiert die Wiederholung der imagoAussage als erneutes Lob der "Auszeichnung und Würde dieser Gnade" (huis gratiae excellentia et dignitas). Der Wertigkeit der Beziehung gibt er den Vorrang vor der Bestimmung: Der erste Platz des Menschen innerhalb der Schöpfung ist schon etwas Grosses, aber bedeutender ist der Adel, "dass er sich der Ähnlichkeit nach zu seinem Bildner gleichsam wie ein Kind zum Vater verhält (quod fictorem suum quasi filius patrem referat). - Hier wird die Beziehungsaussage sogar überzogen, indem sie nach dem im wesentlichen neutestamentlichen Vater-Kind-Verhältnis ausgelegt wird. Aber dieses Verhältnis ist nur ein Zwischenziel der Auslegung: Calvin nähert sich durch sie der qualitativen Aussage: Dem Menschen ist die Herrlichkeit Gottes eingeprägt. Er ist gleichsam lebendiges Bild zweier hervorragender Eigenschaften Gottes: seiner Weisheit und Gerechtigkeit (... gloriam suam imprimendo, ut esset quasi viva divinae sapientiae et justitiae effigies). Übersetzen wir "effigies" weitgehend und umschreibend, heisse es: Der Mensch ist Gestalt gewordene Weisheit und Gerechtigkeit Gottes in der Schöpfung. Ohne Zweifel meint Calvin 
damit stets den Menschen im Urstande, - Es liegt nahe, Calvin hier, ähnlich wie in den Schlüsselsätzen zu 1,26ff ein humanistisch-juridisches Interesse zu unterstellen, zumal vom Kontext her die gennanten Repräsentanz- Eigenschaften sich nicht notwendig ergeben. Ziel der Gesamtexegese zur Stelle ist es dann schliesslich, den Vorwurf einer defektiven Schöpfung abzuweisen und dem Menschen alleine die Schuld an seinem Fall zuzuweisen (alium natura conditum fuisse hominem quam nunc sit factus propria defectionis vitio) (vgl. auch vorher zur Freiheit des Menschen im Urstande: Nec vero liberalius cum eo agere Deus poterat quam illi gloriam suam imprimendo ...). - Im Unterschied zu heutiger Auslegung ${ }^{14}$ interpretiert Calvin "similitudo" in 5,3 nicht als Weitergabe einer Bestimmung, sondern als Weitergabe einer Doppelqualität, sozusagen traduzianistisch, der Naturbeschaffenheit wie der Verkehrtheit und Sündhaftigkeit (refertur quidem ex parte ad primam originem naturae: simul tamen notanda est corruptio et vitiositas: quae ... ad omnes posteros defluxit). Der Akzent lieg auf der Sündhaftigkeit des Fleisches. Was an Seth lobenswert ist, verdankt sich der Gnade des Geistes (secundum carnem natus est ... peccator: deinde renovatus est spiritus gratia). - Calvin findet sich also innerhalb der Schwierigkeiten eines substantiellen Imago-Verständnisses positiv wie negativ zurecht durch Anwendung paulinischer Kategorien (cf. Rom $8,1 \mathrm{ff})$.

\section{Gen 6,12-Gen 9,6}

Hatte im Duktus der Priesterschrift im Geschlechtsregister Gen 5 die Abnahme des Lebensalters den "Bruch" in der guten Schöpfung angedeutet, so stellt Gen 6,12 in lehrhafter Weise die Universalitär der Sünde fest. Diese Deklaration des geschehenen Sündenfalls in der Priesterschrift kann aus den genannten Gründen von Calvin so nicht nachvollzogen werden, aber zutreffend betont er die Allgemeinheit der Sünde, indem er "Fleisch" hier nicht als partitive anthropologische Bezeichnung versteht, als Sündigsein des Menschen, sondern als Kollektivebegriff, an dessen Stelle auch "Menschheit" stehen könnte (carnis ... nomen non in malam partem ... sed ... pro hominibus accipitur).

In der Auslegung von Gen 9,6b kommt Calvin der Beziehungsund der Bestimmungsinterpretation der Imago sehr nahe, aber er interpretiert sie durch qualitative Aussagen: In der Verletzung des Bildes Gottes wird Gott selbst verletzt (violari se existimat in eorum persona). Gott hat auch trotz der Sündigkeit des Menschen sein Ziel mit dem Menschen, wie es in der ersten Schöpfung angelegt ist, vor Augen (... ipsum coelestem fictorem, utcunque corruptus sit homo, finem tamen primae creationis habere ante oculos). Auch die von der 
Sündenlehre bestimmte Negativaussage, die Menschen hätten nichts, wodurch sie sich mit Gott versöhnen könnten, scheint der Beziehungs- und Bestimmungsdefinition den Vorrang zu geben (... quum nihil proprium habeant homines unde sibi conoilient Dei gratiam), doch die Fortsetzung lenkt in die Richtung des Qualitativen: sua in illis dona respicit. Wie die Menschen Gottes Gaben aufweisen, so kann auch von ihnen gesagt werden, dass sie das Bild Gottes eingemeisselt tragen (AHSNR schwächer: "in sich tragen): ferunt imaginem Dei insculptam. Allerdings wird diese massive Festlegung auf eine Relation hin aufgelöst: Durch seine Gaben in den Menschen wird Gott zur Liebe und Fürsorge ihnen gegenüber provoziert (sua in illis dona ... quibus provocetur in eorum amorem et curam). Aus den Zwiespalt zwischen finalem und qualitativem Denken windet sich Calvin mit einer quantitativen Restangabe: Von dem Bild Gottes ist nach dem Sündenfall ein Rest übriggeblieben (manere adhuc aliquid residuum). So zeichnet sich der Mensch durch eine beträchtliche Würde aus; sie bestimmt auch seinen Vorrang vor den übrigen Lebewesen (praestet non parva dignitate; qua excellentia eos dignatus est prae animantibus reliquis). ${ }^{15}$ - Wer sich nicht völlig zum dynamischen Bestimmungsansatz durchringt, wird wie Calvin mit positiven und negativen qualitativen und sogar quantitativen Ungenauigkeiten (aliquid!) ontologischer Provenienz seine Auslegung belasten und sich der spätmittelalterlich bestimmten Polemik nur schwer erwehren können. Die Würde des Menschen bleibt letztlich eine eingeprägte, an den Gaben, am Rest der Gottenbenbildlichkeit o.ä. - zumindest von Gott selbst ablesbare. Es ist dann nicht Gottes ausschliesslich synthetisches Urteil, das die Bestimmung seines Ebenbildes durchhält.

\section{2 Kor 4,4; Kol 1,15}

Es ist erstaunlich, dass Calvin in den christologischen Imago-DeiAussagen die Beziehungs- und Bestimmungsdefinition nicht nur voll ausschöpft, sondern für wichtiger hält das die Wesensqualifikation: Zu 2. Kor. 4,4: Mit "Ebenbild Gottes" ist bei Christus "nicht nur sein Wesen gemeint, weil er mit dem Vater 'eines Wesens' ist, sondern auch seine Beziehung zu uns, weil er uns den Vater darstellt" (... quum Christus imago vocatur Dei invisibilis, id non tantum de essentia accipitur, quia sit coessentialis patri ..., sed magis refertus ad nos, quia patrem nobis repraesentat). Die Analogie der Beziehungen kommt voll zur Geltung: Wie der Vater sich im Sohn erschliesst, so erschliesst uns der Sohn den Vater. Repräsentanz bedeutet also Offenbarungsbestimmung der Imago (Exhibit autem se nobis per filium et se quodammodo facit visibilem ... qualiter nobis eius imago, dum quod absconditum alioqui in ipso erat, nobis manifestat - eine Auflö- 
sung des "quod" versücht Calvin nicht - ... Itaque nomen imaginis relationem ad nos habet...).

$\mathrm{Zu} \mathrm{Kol} \mathrm{1,15:} \mathrm{Diese} \mathrm{Schlüsselstelle} \mathrm{nutzt} \mathrm{Calvin} \mathrm{noch} \mathrm{betonter} \mathrm{als}$ die vorige, um den Vorrang einer christologischen Offenbarungstheologie vor einer ontologischen Theologie aufzuweisen. Die in Kol 1,15 wirklich akzentuierte Einheit von Bild und Identität wird nicht übergangen, ihre Ausschliesslichkeit in Christus festgestellt, aber auf ihr liegt das Primärinteresse Calvins nicht: Imaginem Dei invisibilis appellat: quo significat eum solum esse, per quem Deus ... nobis manifestatur. Calvin wirft den Alten - gemeint ist die altkirchliche Orthodoxie - vor, sie hätten bei ihrem antiarianischen Interesse an der Homoousie von Vater und Sohn über das Wichtigste geschwiegen, nämlich quomodo Pater in Christo se nobis cognoscendum exhibeat. Um den Repräsentanz-, also Bestimmungscharakter von "Bild" zu sichern, zieht Calvin hier auch eine anthropologische Aussage des NT heran: Der Mann ist Bild und Abglanz Gottes (vir est imago et gloria Dei), 1 Kor 11,7. - Im Unterschied zu den im Gen -Kommentar versuchten essentiellen und qualitativen Bestimmungen besteht Calvin im christologischen Kontext ausdrücklich auf dem Relationsdenken: Notandum est nomen Imaginis non praedicari de essentia, sed habere ad nos relationem. Das, was die Voraussetzung für die Repräsentanz ist, wird erst im Rückschlussverfahren erschlossen: ${ }^{16}$ Ideo enim imago Dei Christus, quia Deum ... nobis facit visibilem ... inde etiam colligitur homousia, neque enim Deum vere Christus repraesentaret, nisi Verbum esset Dei essentiale. - Bei der Relation muss noëtisch und darum auch hermeneutisch begonnen werden, statt auf dem ontologischen Dogma zu beharren: ... tamen ab illa relatione ... incipiendum; non insistendum in sola essentia. Auch die Selbsterschliessung Gottes in Christus zugunsten des Menschen vermittelt dem Menschen nicht die Vollkommenheiten Gottes, denn der Mensch ist nicht qualifiziert, ihr Offenbarer zu sein. Er hat nur die Fähigkeit, im Spiegel Christi diese Vollkommenheiten zu betrachten: Gottes Gerechtigkeit, Güte, Weisheit und Kraft. Denoch hat er in diesem Spiegel nicht mit etwas von Gott, sondern mit ihm selbst und mit ihm ganz zu tun (... agitur de perfecta Dei sapienta, bonitate iustitia et potentia: quibus repraesentandis nulla creatura sufficerent ... - Deum ... revelari ... nobis in solo Christo et eum tanquam in speculo contemplemur. Nam in Christo suam iustitiam, bonitatem, sapientiam, virtutem, se denique totum nobis exhibet). - Die A-Seität Gottes, seine Freiheit, steht fest, doch sie ist nicht das Thema der Imago-Dei-Lehre, vor allem nicht das ihrer christologischen Erschliessung, sondern das grosse pro nibis der $\mathrm{Zu}-$ wendung Gottes: ... Deum in se ... in nuda sua maiestate, esse invisibilem ... corporeis oculis ... humanis etiam mentibus ... Neque enim quid in se ipso sit, hic tractatur, sed quid in aliis efficiat). 
Zusammenfassed lässt sich zu diesen beiden neutestamentlichen Kernstellen der Imago-Dei-Lehre feststellen: Ihr Ziel ist, obwohl sie "nur" Christologisches auszusagen scheinen, in der Auslegung Calvins die Menschenwürde: Der Mensch wird gewürdigt, die Repräsen$\operatorname{tanz}$ des unsichtbaren Gottes in Christus zu erkennen und sie zugleich als Zuwendung seiner Volkommenheiten zu erfahren. Dabei bleibt die Freiheit Gottes gewahrt, seine Personalität in Beziehung und geschichtlicher Bestimmung. In dieser ontischen Interpretation der Imago Dei in Christo als "Deus pro nobis" befindet sich Calvin auf der Höhe reformatorischer Theologie.

\section{E. 1 Kor 11,7}

Calvin erkennt den vorwiegend inner-anthropologischen Begriffsgebrauch von imago im Kontext des Paulus und scheidet ihn klar von dem der urtümlichen und gültigen Bestimmung. In der Schöpfungsbestimmung besteht Gleichrangigkeit von Mann und Frau, ebenso in ihrer Erlösungsbestimmung (Creatus ... est uterque sexus ad imaginem Dei: neque minus feminas quam masculus ad illam imaginem reformari iubet Paulus). Substantielle Erwägungen sind solcher Hauptbestimmung untergeordnet. Sie erschliessen sich via negationis: ... imago, de qua nunc loquitur ... pertinet ad praesentem vitam, non autem in conscientia sita est. Oder: non hic tractari de innocentia et sanctitate quae peraeque mulieribus ac viris convenit. Also auch in der Heiligung als ethischem Ziel der imago-Bestimmung kommt Mann und Frau Gleichrangigkeit zu. Dennoch folgt Calvin - wie die Kirche in Jahrhunderten vor ihm und nach ihm dem pragmatisch-polemischen hierarchischen Stufendenken des Paulus: Das Herrschaftsmoment wird aus der imago-Wirklichkeit isoliert; dort besteht als Zeichen der Herrschaft Gottes noch einmal Herrschaft des Mannes über die Frau: In hoc superiore dignitatis gradu conspicitur Dei gloria, sicuti relucet in omni principatu. - Die analogische dialektische Einschränkung, die Paulus folgen lässt: Wie der Mann der Abglanz Gottes sei, so sei die Frau der Abglanz des Mannes, bezieht Calvin nicht nur auf die eheliche Zuordnung, sondern versteht sie bewusst universalistisch als geschichtliche Anordnung Gottes auf alle Frauen: Sie sind der - von Gott gegebene (Gen. obj.) - ausgezeichnete Schmuck, d.h. die Würde der Frau wird eigens an Gott selbst zurückgebunden (. . . coronam esse mariti sui, id de universo sexu verum est, si Dei ordinationem respicimus ... docens ideo conditam esse mulierem, ut insigne sit Dei ornamentum). Es ist nicht auszuschliessen, dass sich in dieser Auslegung eine biblische Begründung des neuen humanistischen Lebensgefühls äussert, das sich mit dem reformatorischen verband und die evangeli- 
schen Frauen zu wesentlichen, auch geistigen und kulturellen Mitträgerinnen der Reformation machte.

\section{F. 1 Kor 15,49; 2 Kor 3,18; Kol 3,10; Röm 8,29}

In den aufgeführten Texten sind jeweils christologische und anthropologische Aussagen miteinander verbunden. Darum sind sie in einer Gruppe zusammengefasst:

Zu 1 Kor 15,49: Zunächst erkennt Calvin historisch-kritisch klar den futurisch-eschatologischen Zusammenhang: Es handelt sich nicht um eine paränetische Intention, die Paulus hier vertritt, sondern um eine auf das zukünftige himmlische Leben gerichtete. Doch verleitet das paulinische phorein Calvin zu habituellen und quantitativen Deutugen. Der Begriff des Bildes wird ersetzt durch den der Natur, und ausserdem nimmt Calvin die Typologie Adam - Christus aus Röm 5 auf: . . quem ad modum animalis natura, quae in nobis praecedit, imago est Adae: i ita coelesti natura fore nos Christo conformes. Auch die Zwischenstrecke zwischen dem Nicht-mehr und dem Noch-nicht wird substantiell definiert: Das endgültige Gleichgestaltetsein mit Christus wird die Vollendung unserer Wiederherstellung sein (... hoc complementum erit reparationis nostrae). Das Bild Christi, das wir jetzt schon anfangen zu tragen und in das wir jetzt schon mehr und mehr verwandelt werden, besteht in der geistlichen Wiedergeburt (Nunc enim incipimus protare imaginem Christi et in dies magis ac magis in eam transformamur (sic!): . . ea imago in regeneratione spirituali consistit). Calvin spürt jedoch die Zchwierigkeit einer quantitativen und qualitativen Festlegung und wandelt sie um in einen geistlichen Prozess. Damit entzieht er die imago-Wirklichkeit einer anthropologischen Objektivation und entspricht auf seine Weise der Dynamik von Beziehung und Bestimmung.

\section{Zu 2 Kor 3,18}

Dieser doxologische Schluss jenes Kapitels, das christologisch und pneumatologisch die Herrlichkeit des Neuen Bundes rühmt, stellt Calvin wie alle Ausleger vor die Zchwierigkeit, Relations- und Bestimmungskategorien durch Prozess- und Wandlungskategorien ausdrücken zu müssen. Die Zchwierigkeit ist im Sprachgebrauch des Paulus vorgegeben durch die Begriffe katoptrizesthai und metamorphousthai. Die angemessene Zuordnung dieser Verben wird wohl das erste als das beherrschende und das zweite als das zugeordnete ansehen müssen. So übersetzt z.B. der revidierte LutherText von 1956 metamorphoumetha nicht: "wir werden verwandelt", sondern "wir werden verklärt". D.h. die Doxa geht von dem erhöhten Herrn durch das Evangelium aus in der Wirksamkeit des Heili- 
gen Geistes. In diesem Geschehen treten wir in zunehmendem Masse aus dem Dunkel heraus und werden in zunehmendem Masse ins Licht Jesu Christi gestellt, gleichsam als Reflektoren seiner Herrlichkeit. Eine Reihe moderner technischer Vergleiche böte sich an zur Interpretation dieses Geschehens. - Calvin arbeitet nun in der Deutung des Bildverständnisses nicht, wie es sich anbietet und wie er es im übrigen häufig übt, mit der Vorstellung des Spiegels. Er zerlegt ein Geschehen, Lichtglanz und dessen Abglanz, in zwei Takte: Leuchten des Evangeliums im Heiligen Geist und seine sukzessiv umwandelnde Wirkung in uns. Dabei entzieht er partiell dem Substanzdenken nicht, und zwar via positionis und via negationis. - Die Kraft des Evangeliums: in evangelio nos habere apartam Dei revelationem ... Illic enim Deus faciem suam conspicuam exhibet. Das $\mathrm{Zu}-$ sammenwirken von Geist und Wort, die konstruktive reformatorische Sonderlehre Calvins: totam vim evangelii inde pendere, quod spiritus sancti gratia nobis vivificum redditur. Die einzelnen Wendungen, durch die Calvin die imago-Wirklichkeit ausdrückt: Die Offenbarung des Evangeliums dient nicht der toten Betrachtung, sed nos per eam transformari in Dei imaginem (Der Paulustext spricht von der imago Domini, also Christi); . . . crescendum esse continuo successu tam in notitia quam conformitate imaginis Dei; particula similitudinis (hier von Calvin ebenfalls als Interpretament für eikon verwandt) ... non ad notandam improprietatem posita est; se ad modum exprimendum; ... hunc esse finem evangelii, ut Dei imago, quae inducta ${ }^{17}$ erat per peccatum, reparetur in nobis ... huius instanrationis progressionem tota vita esse continuam: quia paulatim gloriam in nobis suam illustrat Deus. - Wieder begegnen wir der Methode Calvins, die restlichen Substanzkategorien aufzufangen mit eschatologischen Prozesskategorien in der Unterscheidung zwischen Fortschritt schon jetzt und Vollendung erst dann. Die zunehmende Imago-Qualität der Christen ist gleichsam Durchgangsstation ihrer späteren himmlischen Vollkommenheit. $\mathrm{Zu}$ beachten, dass Calvin zwischendurch auch Relations- und Finalkategorien verwendet, welche die Wachstums- und Fortschrittskategorien interpretieren: notitia, conformitas imaginis. Similitudo als modus exprimendus. - Wieweit wir bei diesem intensiven Suchen mit einer Orientierung am geschichtlichen Denken des Humanismus zu tun haben, das dem heilsgeschichtlichen Ansatz des Paulus dienstbar gemacht wird, ist schwer zu entscheiden. Weitaus klarer lasst sich ein isoliertes anthropologisches Interesse ausschliessen. Calvin betont die theozentrische Bedingtheit der Herrlichkeit des Neuen Bundes so stark, dass eher eine Dämpfung der Doxa des neuen Menschen daraus resultiert, immer schon im Ausblick auf 2 Kor 4,7 ff, die dialektische Gegenseite dieser Herrlichkeit, den "Schatz in irdenen Gefässen". 
Auch bei der Auslegung dieser Stelle, welche die Wirklichkeit des neuen Menschen bezeugt, finden wir zwischen qualitativ-substantiellem Denken und Beziehungskategorien konkurrierende Aussagen Calvins. Qualitativ-substantiell vermag er die Erneuerung zum Bilde Gottes folgendermassen zu definieren: Imago autem Dei in tota anima residet, quum non ratio tantum, sed voluntas enim recta est. Das Bild Gottes gestimmt Calvin hier (1548) in einem teilweisen Vorgriff auf seine spätere Auslegung von Gen 1,27 (1560): Dei imago nempe totius animae rectitudo et integritas. ${ }^{18}$ Auch die Schlussbestimmung: haec sit nostra perfectio et beatitas, imaginem Dei gestare (= tragen) gehört in diese kategorie. - Beziehungskategorien begegnen in folgenden Formulierungen: ... finis sit regenerationis nostrae ... ut Deo reddamur similes, ac in nobis reluccat eius gloria ... ita ut homo sapientiam Dei, iustitiam, et bonitatem quasi speculum repraesentet. Allerdings sind in der letztgenannten Bestimmung doch Vollkommenheiten Gottes zum Inhalt der Repräsentanz gemacht. - Sicherlich hebt Calvin in dieser Auslegung auch auf formale Seinsstrukturen ab: ratio, voluntas, sapientia, iustitia, bonitas. Sie sind jedoch, vor allem im Vorrang des voluntaristischen Elements, ebenso aus seinem Augustinstudium, z.B. aus Augustins psychologisierender Trinitätslehre und ihrem anthropologischen konsequenzen, wie aus humanistischem Sprachgebrauch abzuleiten.

\section{$Z u$ Röm 8,29}

Calvin setzt hier gleich: Christi imaginem gestare und conformis esse imagini Christi. Er unterscheidet diese Qualität vom conformis esse Christo. Die Zielbestimmung und die Unterscheidung der Erwählten von Christus werden durch den Imago-Begriff gewahrt, aber dennoch wird die verordnung Gottes eigenartig synergistisch unterlaufen: ut doceret vivum et conspicuum exemplum exstare in Christo, quod omnibus Dei filiis ed imitationem proponitur. Diese Auslegung in Calvins frühestem Kommentar (1539) bleibt sowohl hinter dem inhaltlichen Passiv des Textes (prohorisen symmorphous) als auch hinter der reformatorischen Rechtfertigungs- und Heiligungslehre zurück. Sie ist hinsichtlich ihrer Herkunft sowohl dem Erbe der Devotio moderna wie auch dem humanistischen zuzuordnen. (Die Übersetzung der AHSNR: "So steht der lebendige Christus als Urbild aller Kinder Gottes anschaulich vor uns" glättet hier allzu sehr zugunsten Calvins.) Die Fortsetzung liegt auf der gleichen Linie: nemo coelorum haeres esse potest qui non ante unigenito Dei filio fuerit conformis ... praerogativam obtinet inter omnes filios Dei Christus, merito nobis in exemplar esse datum. ${ }^{19}$ - Doch der Schluss der Inter- 
pretation erreicht, in bonam partem gedeutet, letzlich noch die Textintention und das paulinisch-reformatorische Denken: ... coelestis pater ius et dignitatem, quam filio suo detulit ... vult omnes, qui in haereditatem regni sui adoptat, eius exemplo conformes fieri. Die positive Deutung hängt davon ab, ob einmal die Willenskundgabe des Vaters als seine Selbstverpflichtung verstanden und zum anderen "exemplum" mit "Bild" (so AHSNR a.a.o.) und - zur Unterscheidung - "fieri" noch passivisch fubersetzt wird.

\section{Zusammenfassung}

Aus zeitlichen und räumlichen Gründen ist es nicht mehr möglich zu verfolgen, in welcher Weise die behandelte Auslegung in die Institutio eingegangen ist. ${ }^{20}$

1. Grundsätzlich zeigt das Verständnis der Imarọ Deı in der Exegese Calvins die gleichen Stärken und Schw žc an wie ir der auf ihr beruhende Lehre der Institutio.

2. Generell kann davon ausgegangen werden, ass die Früchte seiner 20-jährigen exegetischen Arbeit von Calvin auch hinsichtlich unseres thematischen Zentralbegriffs in die Institutio einbezogen worden sind.

3. Die Stärke des Imago-Dei-Verständnisses Calvins liegt in ihrer im wesentlichen ontisch-christologischen Orientierung. Urstands- und Endstandsaussagen werden von daher im Rückschluss bzw. im Ausblick auf die Vollendung gewonnen.

4. In den christologischen Interpretationen der Imago erreicht Calvin alle jene Beziehungs- und Bestimmungsaussagen, die nach unserer Erkenntnis auch schon in der priesterschriftlichen Begrifflichkeit angelegt sind.

5. Das dialektische Prozessdenken bewahrt Calvin vor einer einseitigen Objektivation von Qualifikations-, Substanz- und Habitus-Kategorien. Es hält sugleich die futurisch-eschatologische Spannung der Imago-Dei-Bestimmung durch.

6. Generell bedeutet ebenso die theozentrische, am Vorrang der Offenbarung, des Wirkens Christi durch Wort und Geist orientierte Theologie Calvins eine Sicherung gegen die Gefahr einer Vorherrschaft inadäquaten anthropozentrischen Substanzdenkens.

7. Die Zchwäche des Imago-Dei-Verständnisses Calvins sollte nur innerhalb dieses positiven Rahmens bestimmt werden. Sie besteht allgemein darin, dass Calvin vor allem in der Bestimmung des Urstandes von idealtypischen formalen Seinsstrukturen nicht loskommt, die den Sitz der Imago im Menschen fixieren. Für die Charakterisierung des gefallenen Menschen sieht das ein 
Denken in Restkategorien nach sich, das schon sprachlogisch quantitativ orientiert ist.

8. Auch der neue Mensch kann statt als Hörender, der der Bestimmung Gottes entspricht, eher als Repräsentant besonderer Vollkommenheiten Gottes vorgestellt werden. In der frühen Auslogung von Röm 8,29 stören sogar synergistische Züge einer auf Christus bezogenen exemplum- und imitatio-Ethik.

9. Wie anthropologisch bei Calvin die Repräsentanzwürde des Herrschaftsauftrages nur ein Akzidenz ist, da das Bildsein ja im Menschen liegt, so ist christologisch die Identität Christi mit dem Bilde Gottes vorwiegend als Voraussetzung für die Offenbarung von Bedeutung. Der Deutungsbegriff des "Spiegels" für die Imago ist christologisch wie anthropologisch zwar der Beziehungs- und Bestimmungswirklichkeit nahe, bleigt aber stärker dem vorwiegend doxologischen Bereich verpflichtet als der dynamischere des Mandatars, dem Hören und Entsprechen zugeordnet sind.

10. Wie weit die nicht order nicht primär theologisch bedingten anthropologischen Kategorien Calvins eindeutig aus humanistischer Ausbildung order Orientierung stammen, lässt sich wegen des Fehlens mancher planmässig angelegter Hilfsmittel und Vorarbeiten zum französischen Humanismus nur schwer feststellen. In erster Linie scheint ein begrenztes Interesse an formalen Seinsstrukturen, vornehmlich zur Beschreibung des Urstandes in jene Abhängigkeit zu weisen, soweit es nicht direkt durch die Lektüre antiker oder patristischer Texte vermittelt ist. Die Absicht, die spätscholastische Begrifflichkeit hinter sich zu lassen, ist Calvin weitgehend gelungen. Nicht unterschätzt werden sollte Calvins überragende philologische Fähigkeit und seine begnadete Originalität.

\section{Anmerkungen}

1. vgl. meinen Aufsatz: Der Mensch - Bild Gottes, in: Theologia Viatorum Bd 3/Nr. 2 Journal of the Faculty of Theology University of the North 1975.

2. Calvins Lehre vom Menschen, Zollikon-Zürich 1951 Übers. d. engl. Fassung: Calvin's Doctrine of Man, London 1949.

3. Das Grundverständnis der Theologie Calvins. Unter Einbeziehung ihrer geschichtlichen Abhängigkeiten, Beiträge zur Geschichte und Lehre der Reformierten Kirche, Bd. 15, Neukirchen 1963.

4. Calvin et l'Humanisme, Cahiers de la Revue d'Histoire et de Philosophie Religieuses, Nr. 45, Paris 1976 - Für eine ausführliche Darstellung des Themas, die sich nicht auf die direkte exegetische Überprüfung beschränken muss, wären noch heranzuziehen: J L Schmidt. A comparative study of the doctrine of man in Sigmund Freud and John Calvin, Pittsburgh, 1959. 119/1. Thesis - Xenia Theological Seminary Pittsburg - R McAlpine, The anthropology of Calvin and Brunner: a comparison, Richmond, Va., 1940. Thesis - Union Theological Seminary, Richmond, Va. - J Faber, Imago Dei bij Calvijn, Calvijns Leer over de mens als beeld God krachtens de schepping. (Lucerna, 1:5-32, 1960) - B Hall, Calvin and 
Biblical humanism (Hugenot Society of London, Proceedings, 20:1959-209, 1961 Ph. Liard, L'anthropologie de Calvin; recherche des themes, de leur sources et essai de critique. Montpellier 1963. 1811 Thesis - Faculté libre de theologie protestante.

G Bockwoldt, Das Menschenbild Calvins (Neue Zeitschrift für systematische Theologie und Religionsphilosophie, 19 (2), 170-189, 1968.

$\mathbf{R}$ Prins, The Image of God in Adam and the restoration of man in Jesus Christ; a study in Calvin. Scottish journal of theology, 25: 32-44, 1972.

Die Ergebnisse meiner Auslegung, gvl. o. Anm. 1, die ich als Massstab für die Überprüfung der Auslegung Calvins nehme, seien nach dem Summary am Schluss des gen. Aufsatzes wiedergegeben (a.a.O. S. 109 ff):

\section{Calvyn se Antropologie}

\section{Samevatting}

Omrede beperkte tyd en ruimte is dit nie meer moontlik om vas te stel op watter wyse die uitleg in die Institutio tereggekom het nie.

1. Basies vertoon die verstaan van die Imago Dei in die eksegese van Calvyn dieselfde kragte en swakhede as die leer in die Institutio wat op genoemde eksegese berus.

2. In die algemeen kan as vertrekpunt geneem word, dat die vrug van sy 20 -jarige eksegetiese werk ook deur Calvyn met betrekking tot die sentrale begrip van ons tema in die Institutio betrek is.

3. Die krag van die Imago-Dei-begrip van Calvyn is geleë in die wesentlike onties - Christologiese oriëntasie. Uitsprake oor die oersituasie en die eindsituasie word daarvandaan deur besluit oftewel in die vooruitsien na die voleinding verkry.

4. In die Christologiese interpretasies van die Imago kom Calvyn by al daardie verhoudings- en bestemmingsuitsprake uit wat o i ook reeds in die spraakgebruik van die priesterskrif aangelê is.

5. Die dialektiese prosesdenke bewaar Calvyn van 'n eensydige objektivering van kwalifikasie-, substansie-, en habituskategorië. Dit behou tegelyk deurgaans die toekomstige-eskatologiese spanning van die Imago-Dei-bestemming.

6. In die algemeen beteken net so die teosentriese teologie van Calvyn, wat aan die openbaring van die werk van Christus deur Woord en Gees georiënteer is, ' $n$ beveiliging teen die gevaar van 'n heerskappy van ontoereikende antroposentriese substansiedenke.

7. Die swakheid van die Imago-Dei-begrip van Calvyn behoort slegs binne hierdie positiewe raamwerk bepaal te word. Dit bestaan in die algemeen daarin dat Calvyn veral in die bepaling van die oersituasie nie van ideaaltipiese formele synstrukture, wat die plek van die Imago in die mens vasstel, loskom nie. Vir die karakterisering van die gevalle mens veroorsaak dit 'n denke 
in reskategorië (oorblyfselkategorië) wat reeds taalkundig kwantitatief georiënteer is.

8. Ook die nuwe mens kan pleks van hoorder, wat ooreenkomstig die bepaling van God is, eerder as verteenwoordiger van besondere volmaakthede van God voorgestel word. In die vroeẽ uitleg van Rom. 8,29 steur selfs sinergistiese trekke 'n exemplum- en imitatio-etiek wat op Christus betrekking het.

9. Soos antropologies by Calvyn die verteenwoordigingswaardigheid van die heerseropdrag slegs ' $n$ toevoegsel is, aangesien die beeld-wees immers in die mens geleë is, so is Christologies die identiteit van Christus met die beeld van God hoofsaaklik as voorwaarde vir die openbaring van betekenis. Die uitleggende begrip van die "spieël" vir die Imago is Christologies sowel as antropologies weliswaar naby aan die verhoudings en bepalingswerklikheid, maar bly sterker verbonde aan die hoofsaaklik doksologiese sfeer aan die meer dinamiese van die mandataris waartoe hoor en beantwoord toegevoeg is.

10. Hoe ver die nie- oftewel die nie primêr, teologies bepaalde antropologiese kategorië van Calvyn onmiskenbaar uit humanistiese opleiding of oriëntasie kom, kan moeilik vasgestel word vanweë die ontbreking van baie planmatig aangelegde hulpmiddele en reeds gedane werkstukke oor die Franse humanisme. Eerstens lyk dit asof 'n begrensde belangstelling in formele synstrukture, vernaamlik by die beskrywing van die oersituasie, op 'n sodanige afhanklikheid wys, in soverre dit nie direk deur die lektuur van antieke of patristiese tekste bemiddel is nie. Calvyn het omvattend daarin geslaag om die laat-skolastiese begrippe agter te laat. Calvyn se uitstaande filologiese vaardigheid en sy begenadigde oorspronklikheid behoort nie onderskat te word nie.

MAN - THE IMAGE OF GOD

\section{A Basic Formula of Jewish-christian Anthropology.}

Introduction.

A theological anthropology does not claim to say everything about man but at least to contribute some fundamentally important things, not from empirical analysis of man but from God's relevation. The encounter between God and man is here described by the formula: man the image of God. This formula belongs both to the Old Testament and to the New and shows how God the creator seeks rebellious man. 


\section{The main Biblical reference for this doctrine.}

1. Gen 1:26-28

2. Exposition. Various explanations have been given for the expression: Let us make man in our image.

a. The trinitarian explanation is an interpretation by the Christian church but does not fit the original text.

b. The author prefers the explanation that the plural form is a plural of majesty which implies a divine self-relevation.

c. The plural of majesty also indicates the boundary between creature (man) and the Creator.

The Hebrew word 'man' is a collective and means: mankind. The direct translation of 'selem' is: statue, plastic copy. This seems to imply that God is not to be compared to animals as many neareastern religions believed. Man is the copy of God.

The second key word 'dmuth' serves to soften the comparison and means 'something like'. The expression as a whole therefore implies: man was created in the true image of God but in the sense that it seemed as if man had this image. The uniqueness of God is carefully protected. The prohibition of graven images in Ex 20 is based on the fact that man is God's representative and image in creation: therefore no other man-made images are required. Man's creation as male and female has no special significance: the same pattern was followed in the case of the animals. It does however show another distinction between man and God: God can have no differentiation of the type man-woman. In contrast to the animals, this differentiation as man and woman is the only subdivision in man's created nature. The power inherent in the image of God is given to man as a gift of God's grace: it is a blessing (vs 28).

\section{Does the OT recognize a loss of the image of God in man?}

1. The Problem.

The problem is that the total loss of the image would make man into a mere animal or even less. On the other hand, if the image is partially retained, it must be shown what has remained of the image and what causes it to be (partially) preserved. These questions have important social implications.

2. The exegesis of Gen 6:12-13, Gen 9 and Gen 17: 1f.

The image of God in man is seen as a relationship that rests on God's initiative: it is man's destiny even though it has been made inactive by $\sin$.

3. A further hermeneutical problem.

The suggestion that the P-text knows no history of the fall and no loss of the image is refuted by the author. 
4. Summary.

(i) The statements about the image of God in man are mainly statements about man's destiny.

(ii) The image of God must not be understood as some substance in man.

(iii) The continuity of the image of God in man even after the fall rests on the faithfulness of God.

(iv) It is not possible to define quantitatively how much of the image has remained and how much has been lost.

(v) From the point of view of faith and from the convenant the image of God may be assumed as the destiny of every man.

III. The image of God according to the New Testament.

Statements of importance are a) christological (2 Cor. 4:4; Col 1:15); b) anthropological (1 Cor 11:7); c) both (1 Cor 15:49; 2 Cor 3:18; Col 3:10; Rom 8:29).

In the NT Christ is the image of God in whom the glory of God appears (2 Cor 4:4; Col 1:15).

An additional difficulty is that Greek and late-Jewish elements of thought are present in the NT view of the image of God.

Similarity with the OT consists in 1) the prohibition of all imageworship; 2) the image of God in man is seen as a relationship; 3 ) the image rests on God's initiative.

Differences consist in the following: 1) a hierarchical presentation of the image: in 1 Cor 11 woman is seen as subject to man; also the mediatorship of Christ between God and man is peculiar to the NT.

The NT presents the following new elements:

1. In Jesus Christ we have more than a man destined to be God's image: He is-the image.

2. Christ's function as image of God exists in His service of God's self-revelation.

3 The image of God in Christ is a sign of God's solidarity with His creation.

4. Without this solidarity extended by God, creation would be darkness. (2 Cor 4:4).

5. Christ as the image of God is entirely different from the first bearer of the image: He is totally free in respect of creation and above it, in no way bound by it.

6. This truth becomes available to man by his accepting the announced Good News, the Gospel.

7. In community with Christ in word, faith and obedience the new creation has already arrived.

8. All men are referred to Christ for true participation in the image of God both in the old and the new creation. 
9. Christian life receives a definite direction: laying down the old way of life, moving toward the consummation in constant renewal.

10. Now and in future the destiny of believers is: to become conformed to the image of Christ (Rom 8:29)

IV. Consequences of the Biblical statements about the Image of God.

1. We must see in every man the image of God. This forbids racial discrimination as well as euthanasia.

2. Man's sexuality is part of his nature as image of God: it is a differentiation within the unity of the image.

3. As God stands in relation to man, so man must also stand in relation to the rest of creation.

4. Modern man should remember from whom he receives his great powers, whose blessing he must await and to whom he is responsible if he is to avoid falling into chaos.

5. Recognition of the image of God leads man to an ethic of self-limitation. This refers to atomic weapons, to pollution, to the comsumption of natural resources, etc.

6. A Christian doctrine of the image of God must be oriented to Jesus Christ from whom we may learn the source of the image as well as our destiny. By remaining in Him and in His discipleship we may live as God's representatives in the technical age.

5. cf. J. Calvins Auslegung der Genesis, Übersetzt $u$, bearbeitet von W. Goeters u. M. Simon, in: Johannes Calvins Auslegung der Heiligen Schrift, Neue Reihe, (=AHSNR), Bd 1, Neukirchen 1956 bzw. Opp. Calv. XXIII, Sp $25 \mathrm{ff}-$

Die neutestamentlichen Textauslegungen werden nach folgenden Calvinausgaben Zitiert:

Röm 8,29 Opp. Calv. XLIX, Sp 160; AHSNR 16, S. 172

1 Kor 11,7 Opp. Calv. XLIX, Sp 476; AHSNR 16, S. 412

1 Kor 15,49 Opp. Calv. XLIX, Sp 560; AHSNR 16, S. 465

2 Kor 3, 18 Opp. Calv. L, Sp 46 f; AHSNR 16, S. 515

2 Kor 4,4 Opp. Calv. L, Sp 51 f; AHSNR 16, S. 518

Kol 1,15 Tholuck-Ausg., S 129 f; AHSNR 17, S. 292 f

Kol 3,10 Tholuck-Sudg., S 156 f; AHSNR 17, S. 331

6. s. EBer, a.a.O., S. 98

7. Ita primaria sedes divinae imaginis in mente et corde fuit

8. sensus omnes ad moderatum rationis obsequium prompti et formati

9. dum mens, voluntas, sensusque omnes divinum ordinem repraesentant

10. cf. EBer, a.a.O., S. $98,100,103$, These 1 
11. partem dignitatis

12. vgl. auch Bultmanns Theologie des Neuen Testaments in der Interpretationenes paulinischen pistis-Verständnisses

13. Eine isolierte Wertung der Priesterschrift ist natürlich bei Calvin nicht vorauszusetzen. - Zum "Bruch" in der Schöpfung nach $P$ vgl. II, 1,2 = S. 102 meines Aufsatzes

14. s. EBer, a.a.O., S. $102 \mathrm{f}$

15. zur Kritik, s. Eber, a.a.O., S. 103 f, Thesen $2-4$

16. cf. ähnlich die trinitarische Veranderung der Offenbarungstheologie in Karl Barths Kirchlicher Dogmatik

17. Im Deutschen: "aufgehoben"; "durchgestrichen", AHSNR: "entstellt"

18. vgl. o. zu Gen. 1,26 ff

19. als Variante im lat. Text vorgeschlagen: "debet"

20. Vgl. im I. Buch: 1,1-2; 1,3; besonders I, 15, 3-6 zur Urstandslehre - im II. Buch: 1,1; 2,1 u. 17; 8,51; 12,6 u, 7. - im. III. Buch: 2,1; 2,12; 3,9; 6,1; einen Höhepunkt stellt 7,6 dar. (vgl. meine These IV, 1, a.a.o., S. 107 u. 112); 11,5; 20,40; 25,6 und im IV Buch: 20,24 . 